\title{
(Semantic Web) Services for e-Learning
}

\author{
David E. Millard, Karl Doody, Hugh C. Davis, \\ Lester Gilbert, Yvonne Howard, Feng Tao, Gary Wills \\ School of Electronics and Computer Science \\ University of Southampton, UK \\ \{dem, kad202, hcd, lg3, ymh, ft, gbw\}@ecs.soton.ac.uk
}

\begin{abstract}
Service-Oriented Architectures are becoming a popular system paradigm in e-learning, and there are several efforts to create community driven service frameworks. In this paper, we argue that these efforts could benefit if they also drew on Semantic Web technologies to create (Semantic Web) Services services that use resources described on the semantic web. We present a demonstrator which uses three such services to search over question bank data. We show how the use of Semantic Web technologies not only promotes high-quality and interoperable metadata, but that it also enables reasoning rules to be declared that make developing new services easier.
\end{abstract}

\section{Keywords}

Semantics, e-Learning, Web Services.

\section{INTRODUCTION}

In the last few years there has been an increasing interest within the e-Learning community in ServiceOriented Architectures (SOAs): loosely coupled, distributed sets of components that share common interface standards. The hope is that by concentrating community effort on developing a large range of services, it will become possible to construct e-learning systems more easily and flexibly by orchestrating a number of services to some common purpose (Olivier, 2005; Wilson et al. 2004).

At the same time there has been a push within the Web community towards the Semantic Web, a vision in which formally described data is placed onto the Web for machine, rather than human, consumption (Berners-Lee et al. 2001). Although Web Services are themselves a popular standard, the semantic web is 
more focused on data than process, and technologies such as RDF and OWL have been developed so that applications can share a common interpretation of data ${ }^{1}$.

The services and semantic worlds come together in two ways. The first is that services provide a semantically rich description of their functionality in order for applications to reason about them in the same way as they reason about data on the semantic web. This approach is the one taken by work in the Semantic Grid area (De Roure et al. 2003). We call these Semantic (Web Services). The second (which is complementary to the first) is that the applications that use the data from the semantic web could actually be services themselves. It is this type of component that we call a (Semantic Web) Service.

In this paper we present our work on creating a set of example (Semantic Web) Services in the e-learning domain that demonstrate a range of advantages from working with the Semantic Web. Our contribution is to show how existing Semantic Web technology could be applied to the e-learning domain, in particular how our services are designed to both contribute to the growing pool of e-learning services, and also to demonstrate to the community the advantages of working with semantics as well as service architectures.

In Section 2 we describe the arguments for service-oriented architectures and look at some of the existing work in the area of services for e-learning, including the e-Framework: an architecture for the collaborative development of Web Services. In Section 3 we make the case for semantics in e-learning, and present three clear advantages: well-formed metadata, interoperability, and the capacity for reasoning. We believe there are few examples of the later in the e-Learning domain and in the following Sections we present three question bank services, designed to fit into the e-Framework, that operate on Semantic Web pages and demonstrate the full power of the semantic approach. In Section 4 we present the ontologies we have created that describe the key resources in the question bank area, in Section 5 we present the services themselves, and in Section 6 we describe the Web application that invokes them. We finally conclude in Section 7 by looking at how the services might be extended to further take advantage of the existing semantics, and argue that the Semantic Web and Web Services together form a powerful framework for the future development of e-Learning applications.

\section{SERVICES FOR E-LEARNING}

In the last few years there has been a trend towards Service-Oriented Architectures (SOA) in middleware systems. SOAs are an attempt to modularize large complex systems in such a way as they are composed of independent software components that offer services to one another through well-defined interfaces. The service approach is ideally suited to more loosely coupled systems. Wilson et al. (2004) present the main advantages of using SOA:

- Modularity: In a Service-Oriented Architecture, services are dynamically coupled; this makes it easy to add or replace services, even with a running system.

\footnotetext{
${ }^{1}$ For more information on Semantic Web technologies see the W3C web page: http://www.w3.org/2001/sw/
} 
- Interoperability: Services communicate using standard protocols and are defined using standard specifications. This means that third-party specifications can easily be used with existing service-based systems.

- Extensibility: Because of their modular and interoperable nature, service-based systems can be easily extended, which reduces the danger of technology 'lock-in'.

As e-learning applications become more integrated, and e-learning systems more distributed, there is an increased need to manage their software and data components (Anido et al, 2002). As a result there is interest in applying SOAs to the domain in order to help construct future e-learning applications (Rodriguez et al, 2003; Okamoto and Kayama, 2004).

With SOAs there is a need to design complementary services that can be used together to some end. Sometimes these are known as composite services, but in larger cases can represent the infrastructure for an entire domain. These are known as service frameworks.

In the UK all the Further and Higher Education funding councils finance the Joint Information Systems Committee (JISC). JISC have recently undertaken a programme to develop a common service framework for e-learning, e-research and e-administration, which they call simply The e-Framework (Olivier, 2005).

The e-Framework is a suggested architecture, designed to be populated by the community itself, and whose constituent services are intended to evolve and change over time. To enable this, JISC have funded a number of Reference Model projects, designed as community focus points, databases, and guides for the construction of services in the e-Framework.

The authors are involved in the Framework Reference Model for Assessment (FREMA), a reference model for service-based systems concerned with all aspects of student assessment. As part of this work we have been exploring how e-learning systems could exploit the power of the Semantic Web (Tao et al, 2005). In order to demonstrate this within FREMA we have been developing a number of example services that are a core part of the assessment domain and which build on ontological data defined using Semantic Web technology.

\section{SEMANTICS FOR E-LEARNING}

We believe that the semantic aspects of learning content are the key to facilitating large scale collaboration of e-learning activities over service-oriented infrastructures. This is because of the complexity of learning resources and the ability of semantic meta-data to make some of that complexity machine readable.

In order to use semantics that are shared across a number of applications it is necessary to form a consensus in the domain at the conceptual level. The view in the Semantic Web community is that an ontology is the best vehicle in this context to formally hold a specification (of the conceptualisation) that can be shared within the community to describe semantics accurately and consistently.

Ontology is the study of existence, and an ontology is a description of what is permitted in a given understanding of existence. In practical terms this means that an ontology is a description of what 
concepts and relationships are permitted in a semantic network that describes parts of a given domain. An ontology is therefore similar to a glossary but with richer structure, relationships, and axioms that describe a domain of interest precisely.

These rich semantics offer both teachers and learners new opportunities for locating and reusing resources (Ronchetti and Saini, 2004; Sridharen et al, 2004; Sampson et al. 2004). For developers they offer at least three advantages:

Well-formed metadata: Constructing an ontology forces information designers to consider all of the relationships between all their resources and it also encourages them to think about how to arrange their meta-data classes into hierarchies. In short it encourages designers to consider their meta-data as a whole and consider their requirements more holistically.

Interoperability: If several developers in a domain are using the same ontology to describe their domain then it enables their systems to interoperate much more easily. Even in cases where the ontologies have been only partially agreed, it is still an advantage as it is well understood by all systems which metadata is agreed and which is not - even if the same concepts appear in the different ontologies (this is because classes, attributes, and relationships are given unique names on the Semantic Web).

Reasoning: Formal and explicit semantics allow reasoning rules to be declared and new relationships derived. This could be done programmatically but the formal semantics allow this to be done declaratively and thus processed by generic programs. For example, rules for synonyms could be declared that enable a system to go beyond the limitation of lexical searches to semantic searching: thus a request for myths on Yeti will also yield Abominable Snowman (Carr et al, 2005).

In practice very few Semantic Web systems make use of all three of these advantages. Well-formed metadata is common in Semantic Web applications, and even non-semantic Web systems can use ontology design to help make sure that their meta-data is well considered (Fensel, 2000). Interoperability is often quoted but rarely demonstrated, although it is seen by many as the driving force behind the Semantic Web, others are suspicious of the possibility of agreeing common ontologies between disparate researchers (Marshall and Shipman, 2003). Reasoning seems the most elusive. Perhaps this is becuase developers are used to working programmatically, or maybe it is to do with the availability of tools, which are only just maturing.

For our semantic services we wanted to demonstrate this third advantage of reasoning and show how a set of Semantic Web pages could be reasoned about and used as a resource by Web Services.

\subsection{A Question Bank Scenario}

The purpose of our semantic services is to demonstrate the higher level advantages of using semantics to the e-learning community. For this purpose we wanted to choose services that were core to our domain of e-learning assessment, and therefore chose Item Banks which are not only key applications that have a 
central part within e-Learning, but also have several existing implementations and example datasets. An Item Bank is a repository of learning resources that can be queried across a network. In particular we looked at Item Banks dealing specifically with question items (also known as Question Banks).

One advantage of using Semantic Web and Web services together is that it allows a community to access the resources at different levels depending upon their knowledge and skill. The resources are described as ontological instances and are Web accessible RDF files for those who want to query them directly (the most straightforward paradigm of the Semantic Web). The Web Services and their WSDL descriptions are also publicly accessible if developers wish to interact with that level instead (the paradigm of SOAs). Finally, we developed a client that consumes the Web Services and provides a web front-end for nondevelopers (the traditional paradigm of the human-oriented Web).

In the next section we will present the first of these interfaces, the ontologies that allow questions and syllabi to be described on the Semantic Web.

\section{ONTOLOGIES FOR QUESTION BANKS}

The first part of the information design involves deciding the ontologies that should be created and performing some knowledge acquisition to generate them (Tao et al, 2005).

Figure 1 shows the e-Learning information we chose to model and the three ontologies that we developed to do this.

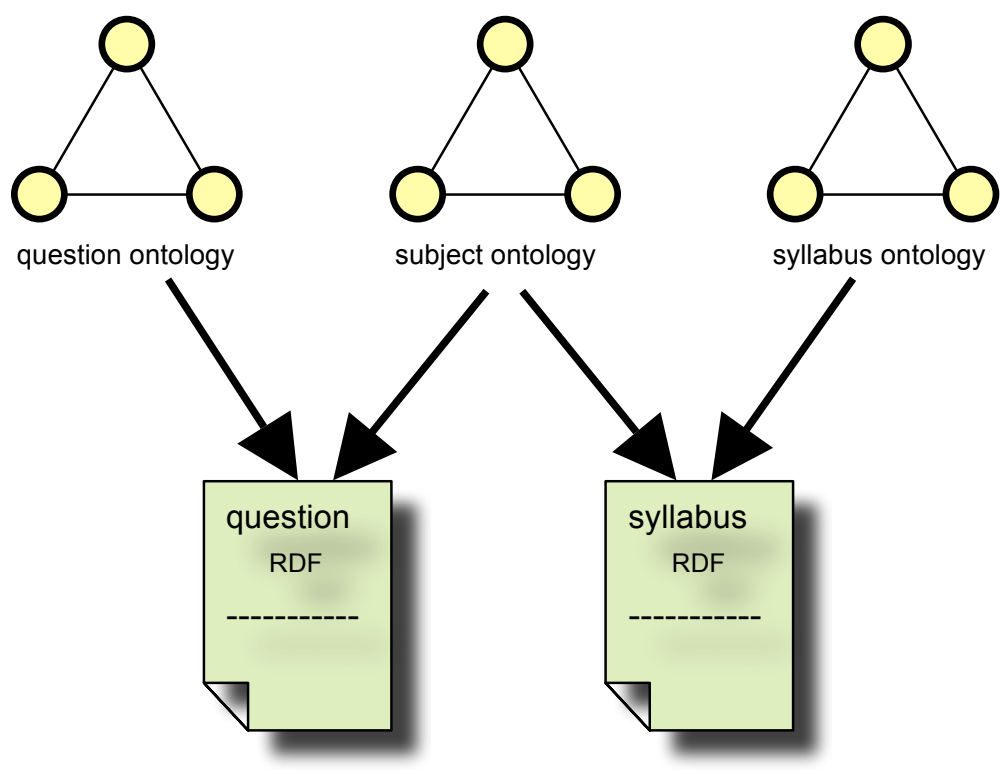

Figure 1: Example Ontologies and RDF documents

The information is contained in two RDF documents. The first contains descriptions of questions, similar to those that would normally be found in a Question Bank. The second contains descriptions of syllabi, as would normally be found in a course prospectus. 
We modelled these two sets of information using three ontologies. The first is a question ontology that describes all the properties of a question's structure and purpose. The second is a subject ontology that describes the key areas within a particular topic that is to be taught and how they are related. The third describes the structure and properties of a syllabus.

Using RDF allows the project to utilize semantic benefits such as inference and the ability to respond to different queries not only with exact question matches, but with other questions that the system feels will be relevant given its knowledge of the domain that is provided by the topic map. In the rest of this section we will look at each of these ontologies in turn.

\subsection{The Question Ontology}

Our knowledge acquisition process for the question ontology was based on existing standards for question metadata. In particular we looked at the E3AN (Electrical and Electronic Engineering Assessment Network) database (White and Davis, 2000) and the IMS QTI (Question \& Test Interoperability) specification $^{2}$.

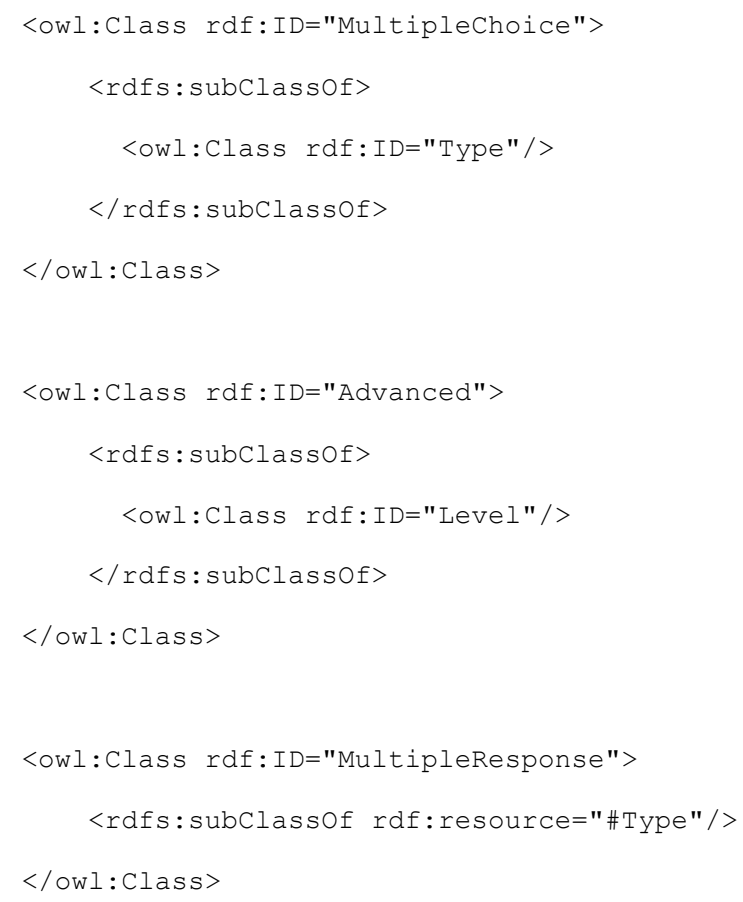

Figure 2: Sample of Ontology Specification in OWL

This also had the advantage that we could convert a number of questions already marked up for the E3AN system into ontological instances with the minimum of manual intervention. 
This has provided us with a large quantity of quality material that would have been unrealistic to produce ourselves; it also means that we have a real world dataset that is representative of actual questions being used by practitioners. The question ontology is described in OWL. Figure 2 shows a short excerpt from the specification that describes some of the classes. The E3AN questions are currently stored in a Microsoft Access database, so we have converted them into the RDF representation. An example of markup for one question is shown in Figure 3. As would be expected, many of the relationships are from the question ontology described above (marked question), however the real power of our system comes from the fact that there are also relationships from the subject ontology (marked skos).

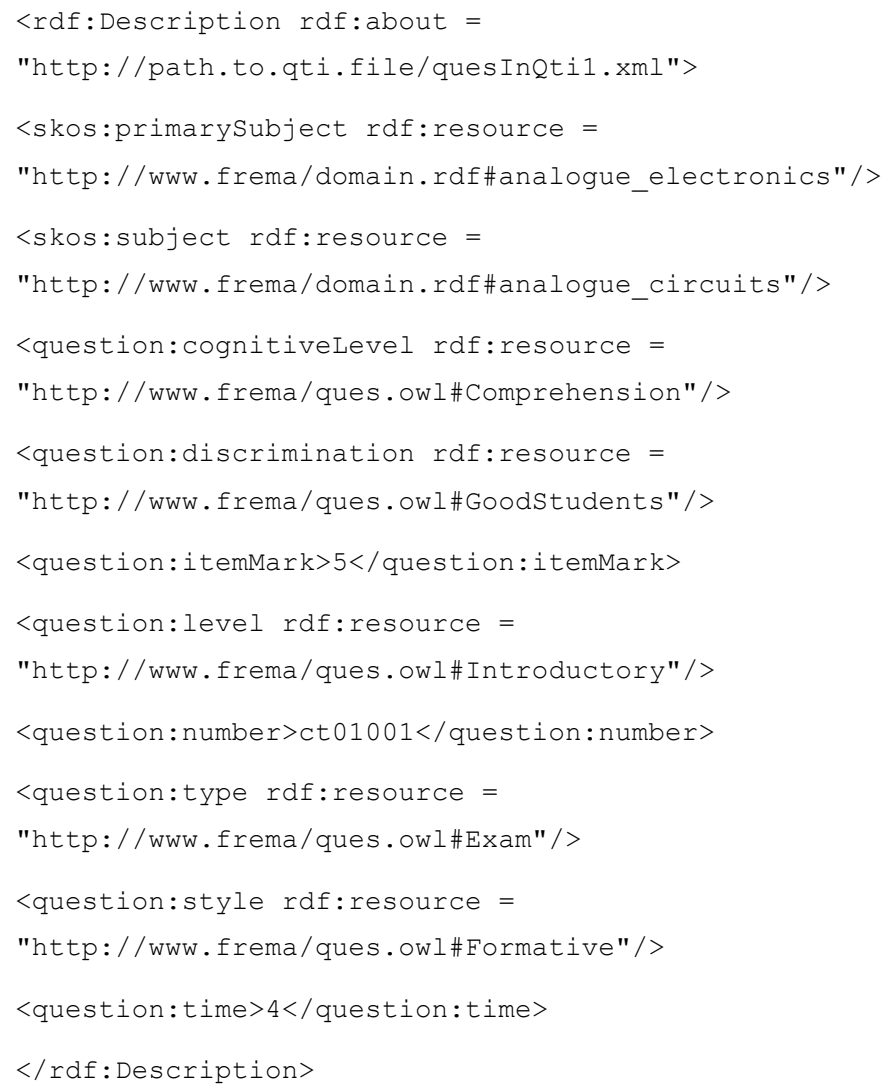

Figure 3: Sample Question in RDF

\footnotetext{
${ }^{2}$ IMS QTI homepage: http://www.imsglobal.org/question/
} 


\subsection{The Subject Ontology}

The subject ontology is a description of the topics in the domain, and how they relate to one another. For our knowledge acquisition process we used Topic Maps (Dicheva and Dichev, 2005), the $\operatorname{SKOS}^{3}$ (Simple Knowledge Organisation System) ontology developed by the W3C for the representation of Topic Maps, and the IEE taxonomy for digital electronics ${ }^{4}$.

Dicheva and Dichev (2005) considered the minimum set of relationships to capture a topic map (minimum in the sense that they still capture most of the important differences between relationships). They examined the SKOS set of relationships and generalised to the following:

- Superclass-Subclass - the relationship between two classes that states that one is the subclass of another

- Class-Instance - the relationship between a class and the instance of that class

- Super-Sub - a generalisation of the various part-whole relationships with asymmetric roles (such as part-of, is-based-on) that are typically used to create hierarchies.

- Relevant-To - a horizontal relation to represent non-hierarchical symmetric relations (such as is-similar-to, is-synonymous-with)

- Mentioned-By - a horizontal relation to represent non-hierarchical asymmetric relations (such as refers-to, discusses, is-used-by)

For our ontology we chose to base our relations on the SKOS ontology guided by these five generalisations. We chose to use the Super-Sub relation, and used the SKOS relation which most closely matched it, the narrower relation.

Figure 4 shows part of the topic map of the digital electronics domain that was constructed in RDF based on the IEE taxonomy. The question instances from E3AN were then annotated with references to the subject instance from the IEE instances that they tested. This process was aided by the fact that the E3AN questions are already categorized using keywords.

We have only used the narrower relationship as our services are intended to function as exemplars of how to leverage semantics and show how reasoning can be used.

\footnotetext{
${ }^{3}$ SKOS Development Page: http://www.w3.org/2004/02/skos/

${ }^{4}$ IEE Authors Guidelines: http://www.iee.org/Publish/Support/Auth/elhier.pdf
} 


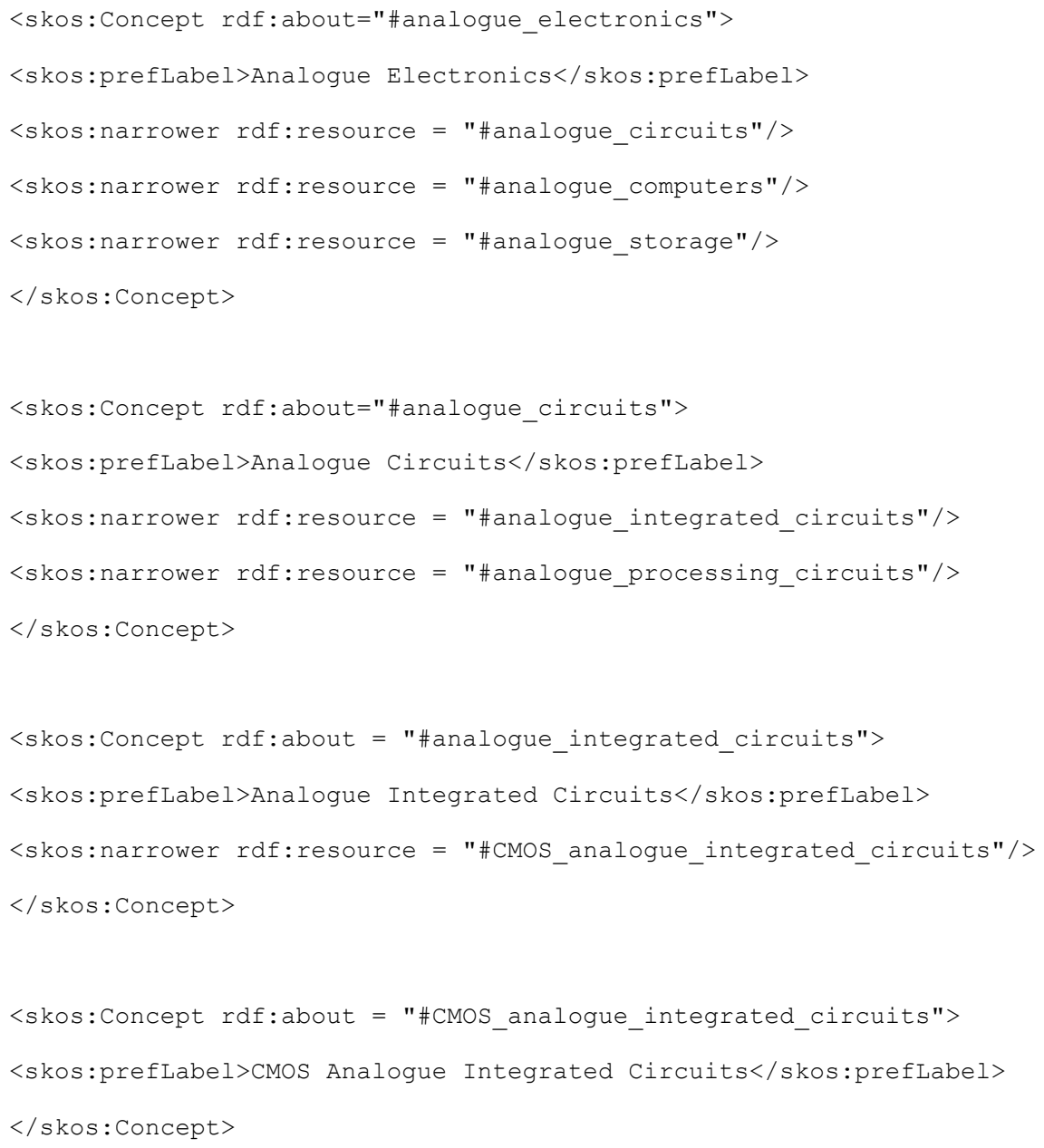

Figure 4: Sample of Ontology Specification in OWL

\subsection{The Syllabus Ontology}

The Syllabus ontology models the key concepts and relations involved in describing courses and their structure. We based our syllabus ontology on the formal module descriptions of the University of Southampton, and identified such details as module weight (number of credits), module staff, and a breakdown on what is taught within that module and in which order.

Although the ordering is part of the syllabus ontology, the subject topics themselves come from the subject ontology described above.

It is because the syllabus ontology and the question ontology share the relations from the subject ontology that they can be reasoned about together. Based on these ontologies and the annotated resources we were 
able to design a number of services that demonstrated how such semantically rich resources might be used by e-learning Web Services.

\section{SEARCH SERVICES}

We wanted to develop several question bank services to show how Semantic Web resources might be used by Web Services. We identified three services that would build upon the use of the ontologies:

$\begin{array}{lll}\text { - } & \text { Basic Search } & \text { (Question) } \\ \text { - } & \text { Similar Search } & \text { (Question+Subject) } \\ \text { - } & \text { Semantic Search } & \text { (Question+Subject+Syllabus) }\end{array}$

These are implemented as Web Services. They are coded in Java, run on Apache Tomcat, and use the Axis framework for deployment. The services use the Jena libraries/API ${ }^{5}$ for ontology/instance querying, and query the RDF files with the RDF Data Query Language (RDQL) ${ }^{6}$.

In addition each service conforms to the WS-I (Web Service Interoperability) standard ${ }^{7}$. This was done by creating conformant WSDL and pursuing a WSDL-first Web Service development approach (which maximizes the interoperability of the Web Service because both the client and service are generated from the same WSDL).

\subsection{Basic Search Service}

The basic search service uses simple attribute/meta-data matching to retrieve a set of questions given a number of fields by the user of the service. This is analogous to the search facilities of many question bank systems such as E3AN (White and Davis, 2000) and TOIA (Technologies for Online Interoperable Assessment) (Sclater et al, 2003). The only difference with this service is that it is directly querying the RDF files on the Semantic Web, using meta-data that has been generated through a knowledge acquisition process.

The service queries the RDF documents using RDQL. An RDQL string is generated from the search parameters and is used to query the question instances. Questions are returned that have the same attribute values as the values given in the search parameters.

The Basic Search Service exposes users to some of the ontological terms directly, and demonstrates the well-formed metadata advantage of Semantic Web resources.

\footnotetext{
${ }^{5}$ Jena Java Framework: http://jena.sourceforge.net/

${ }^{6}$ W3C RDQL page: http://www.w3.org/Submission/RDQL/

${ }^{7}$ Web Service Interoperability Organisation Homepage: http://www.ws-i.org/
} 


\subsection{Similar Search Service}

The second service increases the complexity of the search interface as it takes a question instance as the search parameter. The service then returns other questions that are similar to the one submitted. Although the service is more complex it does not rely on the semantic nature of the meta-data. Instead it uses the metadata heuristically and produces results based on weighted attributes.

The service takes a question identifier as a parameter. It looks up the meta-data for the question with that identifier and uses the results to match this meta-data against the meta-data of every other question.

The similarity of each question to the search question is calculated by summing the weights of all the attributes that match between them. The system then sorts all the matching questions according to their weighting. The system does not return questions that have no matching attributes.

The Similar Search Service relies on additional ontologies being used to annotate the same resource used in the Basic Search Service, drawing on the interoperability advantage of Semantic Web resources.

\subsection{Semantic Search Service}

The final service demonstrates knowledge reuse. This service relies on the annotated syllabus data, which shares some of its ontological markup with the questions (both questions and syllabi are annotated by the subject ontology). Because of this it can use the question data in a search based on a syllabus.

The user specifies the syllabus topics that they are interested in and the system returns a set of questions that address those topics. Reasoning rules encoded in Jena ensure that that topics that are narrower than the target topic still match (i.e. if we are seeking a question on "mobile communication" then those described as "cellular communication" still match).

This not only demonstrates the reuse of the original knowledge, but also the added value that semantics can bring to e-Learning applications. It is much simpler to declare the Jena rules than it would be to programmatically achieve the same effect using a standard query interface.

The semantic search takes a list of syllabus elements as its parameters. Since each syllabus element is mapped onto a subject in the subject ontology, and questions are mapped onto the same subjects, it is possible to use this information to find questions that match this element. The Jena rules mean that it is also possible to find topics close to it in the topic map hierarchy and return these as well. Consider the following two triples:

$\begin{array}{lll}\text { Analogue_electronics } & \text { narrower } & \text { Analogue_circuits } \\ \text { Analogue_circuits } & \text { narrower } & \text { Analogue_integrated_circuits }\end{array}$

They construct this hierarchy:

- Analogue_electronics

○ Analogue_circuits 
- Analogue_integrated_circuits

Using these two triples alone, it cannot be determined whether analogue_electronics relates to analogue_integrated_circuits. But as this is expressed in RDF, we can introduce a transitive rule that states that:

a narrower $\mathrm{b}$ and

b narrower $\mathrm{c}$

$=>$ a narrower $\mathrm{C}$

We can use this to infer extra relations within the topic map. Now when the topic map is queried, analogue_integrated_circuits is known to be a subtopic of analogue_electronics and is known to be two narrower relationships away. The number of relations used to infer the new fact can be used to weight how relevant a topic is to another one and is used by the service to give precedence to closer topics.

If a user of the system requests that they want questions to test a syllabus element that maps to the topic of analogue electronics then questions about analogue circuits will be returned too. Questions about analogue integrated circuits would be returned as well if there were not enough questions returned from the use of the other two topics, but this topic would be weighted more weakly because of its further distance from the topic requested.

Because the Semantic Search Service uses declarative rules to do some of its search work it demonstrates the advantages that Semantic Web resources have in the area of semantic reasoning.

\section{A WEB INTERFACE FOR QUESTION BANKS}

For the front-end of our demonstrator we chose to develop a small website that would allow Web visitors to the page to invoke any one of the three services. The website is written in Ruby, and uses the Ruby on Rails Web application framework. It runs on the Apache Web server.

The website consists of pages with forms that allow the Web Services to be invoked. The Web Services are called with the form data as the method parameters. The returned results are then formatted for display.

Figure 5 shows the architecture of the demonstrator, the different protocols used for communication, and the different RDF resource files drawn on by the search services. 


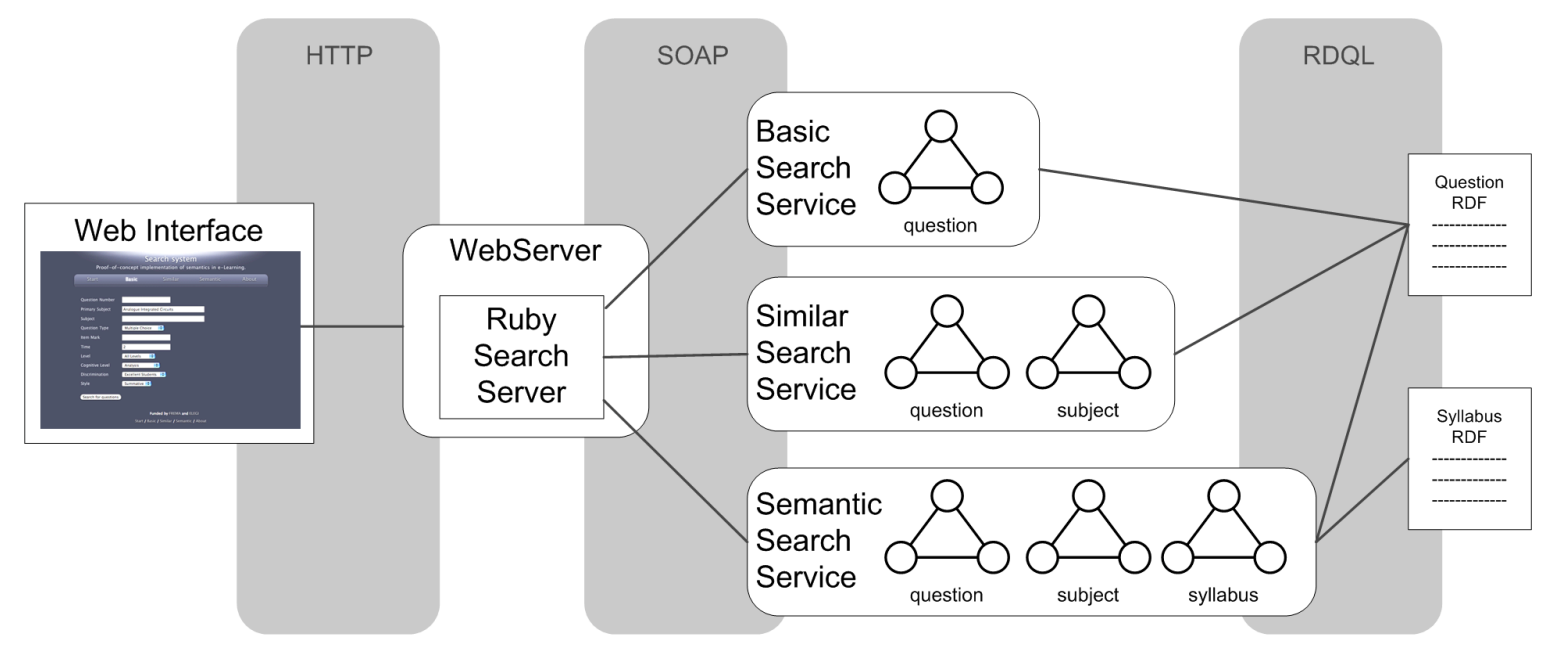

Figure 5: Demonstrator Architecture

Figures 6, 7, and 8 show the query interfaces for the query services (Basic, Similar, and Semantic). The form for the Basic search (Figure 6) supplies input elements for every attribute type. The Similar search (Figure 7) has a single input element for a question number but is normally called via clicking the "Find Similar" link on any question in the result set of a previous search. The form for the Semantic search (Figure 8) provides a syllabus listing with checkboxes. The user ticks the syllabus elements that they want to test and the results page will returns questions matching those syllabus parts.

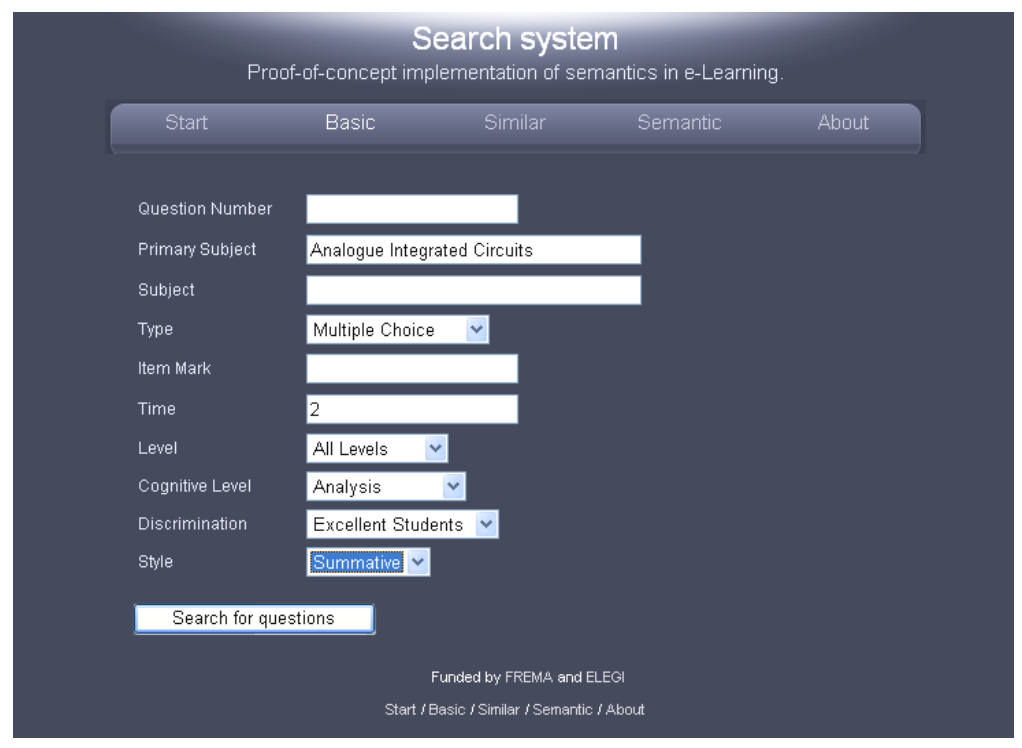

Figure 6: The Basic Search Interfaces 


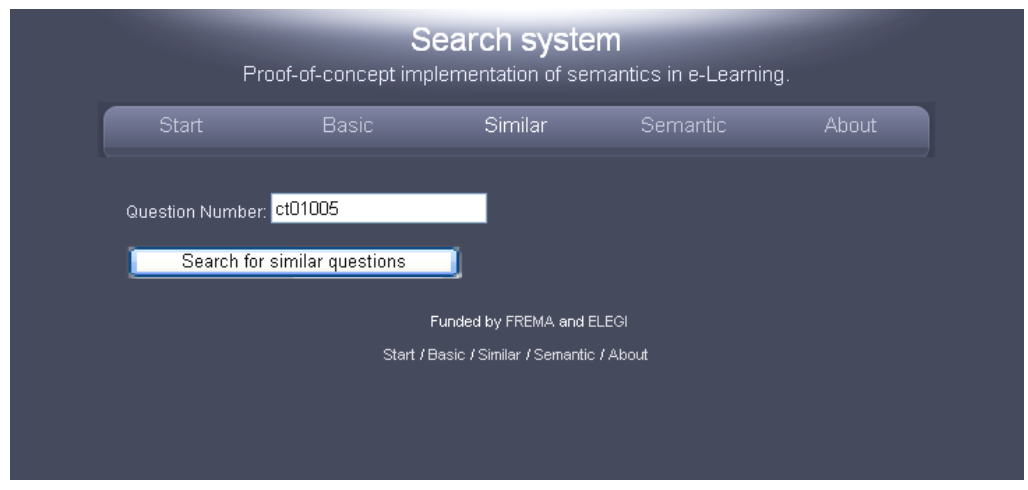

Figure 7: The Similar and Semantic Search Interfaces

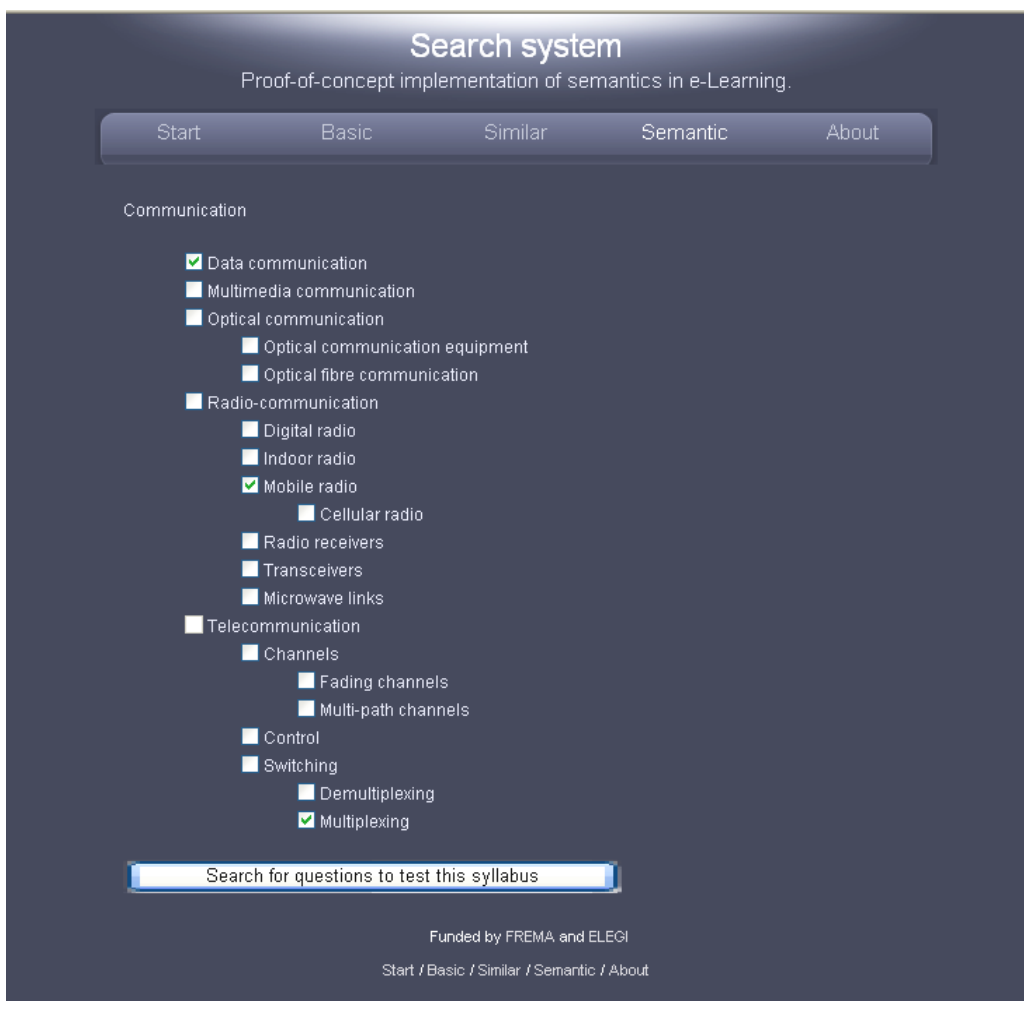

Figure 8: The Semantic Search Interfaces

Because the Web Services themselves had been developed using WS-I, with a WSDL-first approach, it was straightforward to access them from the two different SOAP implementations (Axis for the Java Web Services, and SOAP4R for the Ruby website).

Figure 9 shows the results page returned to the user (whichever service is invoked). A thumbnail from each question is displayed along with some metadata, including the weighting of that result (if appropriate).

The services are integrated in that alongside each result is a link to invoke the similar search service with that question. In this way the services can be used together to locate and explore the question bank. 


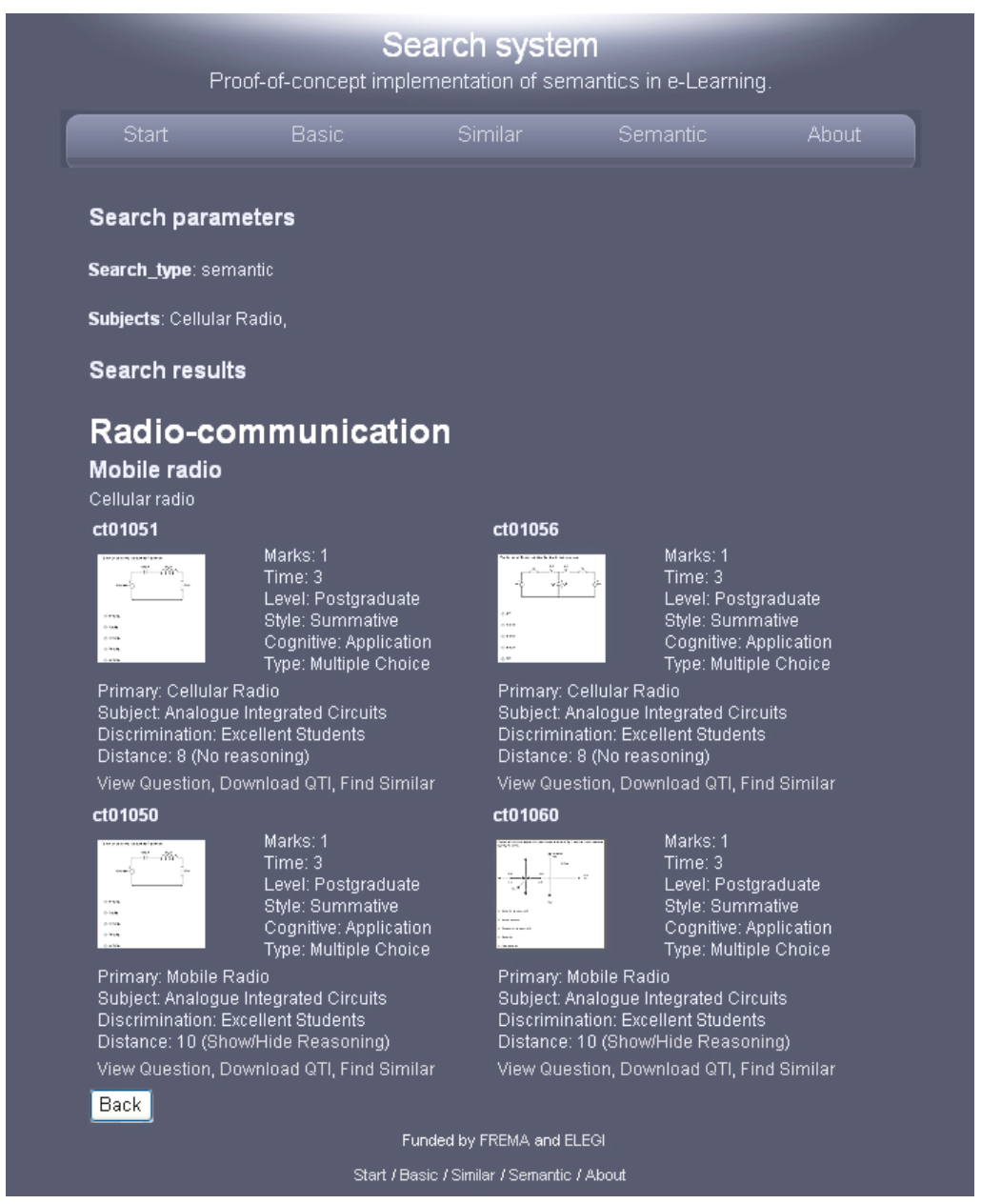

Figure 9: The Results Page

\section{CONCLUSIONS}

In this paper we have described the trend in e-learning systems towards Service-Oriented Architectures and loosely coupled, community driven development frameworks.

We differentiate between "Semantic (Web Services)", which are Web Services described using Semantic Web technology, and "(Semantic Web) Services", which are services designed to use the information on the Semantic Web.

We have argued that in the e-learning scenario the later type of semantic service has much to offer. Not only does it make service development easier but it also makes it possible to mark up resources in such a way that disparate services can access and manipulate them.

We have designed three example ontologies and three Web Services that offer different question bank functionality over resources annotated by those ontologies and described in RDF.

These three services show the three different advantages of using Semantic Web resources: well-formed metadata, interoperability, and reasoning. 
The Basic Search Service uses the RDF resources as meta-data, and takes advantage of the fact that they are described in a consistent way and with high quality, thus demonstrating the well-formed metadata advantage of semantic resources. The Similar Search Service draws on the same resources as the first but with additional annotations from a second ontology, thus demonstrating the interoperability advantages of Semantic Web resources. The Semantic Search Service leverages the semantics of the resources, (and the fact that they share one of their ontologies) by encoding a simple reasoning rule using Jena; thus demonstrating the reasoning advantages of Semantic Web resources.

It would be possible to add further relations to the subject ontology that reflected more of the core topic relationships. For example it is likely that there are some horizontal relationships between subjects that could be modelled by the Relevant-to relation (see Section 4.2). This would allow additional reasoning rules to be applied, possibly across the ontologies. For example:

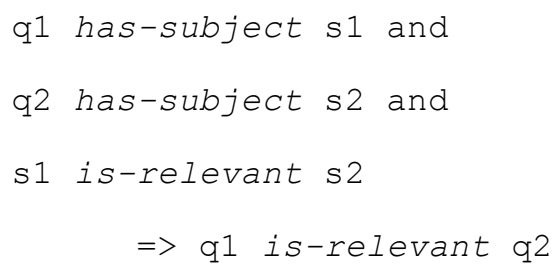

This relation would enable returned questions to be clustered according to the relevancy of their topics. At the moment our demonstrator does not use any information from the syllabus other than the topics. In fact the ordering of the topics is very important in a syllabus, and could be used to help decide how questions might best build on one another.

The work we have presented here is only an example of how the Semantic Web could be coupled with Web Services to help develop e-learning applications. We believe that more complex applications could be very powerful, for example by automatically extracting an appropriate set of questions from a question bank to examine a given syllabus, or by modelling competencies and using that model to build an appropriate test.

We are currently developing a Web Services framework that will enable service developers to create highlevel semantic functionality on top of low-level semantic management services. We hope that this will form a basis for creating a new generation of semantically driven collaborative e-learning services. These would allow learners to semantically annotate their learning experiences with elements such as roles, events, and environments, as well as contribute to the domain knowledge itself in the form of classes, relationships, taxonomies, and rules. This would enable not only the kind of (Semantic Web) Service described in this paper, but also enable learners to exploit the social and learning networks implicitly described in the knowledge base.

Web Services are a useful paradigm for system development in the e-learning domain because of their modularity and flexibility. When coupled with the Semantic Web they promise significant advantages for 
interoperable access, management, and manipulation of the complex resources typically found within the e-learning domain.

\section{ACKNOWLEDGMENTS}

This work has been undertaken as part of the FREMA project, funded under the JISC e-learning programme, and the European ELeGI project, IST-0002205, Sixth Framework.

\section{REFERENCES}

Anido L., Rodriguez J., Caeiro M., Santos J. (2002), 'Towards Standards-driven Educational Content Providers' 2nd IEEE International Conference on Advanced Learning Technologies (ICALT'02), Sept 9-12, Kazan, Russia

Berners-Lee T., Hendler J., and Lassila O. (2001), “The Semantic Web,” Scientific American, May 2001.

Carr, L., Miles-Board, T., Wills, G., Power, G., Bailey, C., Hall, W. and Grange, S. (2004) Extending the Role of Digital Library: Computer Support for Creating Articles. In Proceedings of The Fifteenth ACM Conference on Hypertext and Hypermedia, pp. 12-21, University of California, Santa Cruz, USA.

De Roure, D., Jennings, N. R. and Shadbolt, N. (2003) The Semantic Grid: A future e-Science infrastructure, in Berman, F., Fox, G. and Hey, A. J. G., Eds. Grid Computing - Making the Global Infrastructure a Reality, pages pp. 437-470. John Wiley and Sons Ltd.

Dicheva D., Dichev C. (2005). Authoring Educational Topic Maps: Can We Make It Easier? 5th IEEE International Conference on Advanced Learning Technologies, ICALT 2005, July 5-8, 2005, Kaohsiung, Taiwan, 216-219.

Fensel, D. (2000) “The Semantic Web and Its Languages,” IEEE IntelligentSystems, vol. 15, no. 6, Nov./Dec. 2000, p. $67-73$

Marshall C. C. and Shipman, F. M. (2003). Which semantic web?. In Proceedings of the Fourteenth ACM Conference on Hypertext and Hypermedia (Nottingham, UK, August 26 - 30, 2003). HYPERTEXT '03. ACM Press, New York, NY, 57-66. DOI= http://doi.acm.org/10.1145/900051.900063

Okamoto T. and Kayama M. (2004), "The Learning GRID for Knowledge Building", 4th IEEE International Conference on Advanced Learning Technologies (ICALT'04) August 30 - September 01, Joensuu, Finland

Olivier B. (2005) The Framework: an Overview. JISC.

Rodriguez J., Anido L., Fernandez M. J. (2003), "How can the Web Services Paradigm improve Elearning?" 3rd IEEE International Conference on Advanced Learning Technologies (ICALT'03), July 9-11, Athens, Greece

Ronchetti M. and Saini P. (2004), "Knowledge management in an e-learning system", 4th IEEE International Conference on Advanced Learning Technologies (ICALT'04) August 30 - September 01, Joensuu, Finland, pp. 365-369 
Sampson D. G., Miltiadis D. Lytras, Gerd Wagner and Paloma Diaz (Eds) (2004): "Ontologies and the Semantic Web for E-learning", Special Issue of IEE IFETS Journal of Educational Technology \& Society Special: 2004, Vol. 7, Issue 4

Sclater N., Davis H. C., White S. A., Conole G. C., Danson M. (2003) Technologies for online interoperable assessment Proceedings of the CAL03; (Belfast, Elsevier)

Sridharan B., Tretiakov A., Kinshuk (2004), "Application of Ontology to Knowledge Management in Web based Learning", 4th IEEE International Conference on Advanced Learning Technologies (ICALT'04) August 30 - September 01, Joensuu, Finland, pp 663-665

Tao, F. B., Millard, D., Davis, H. and Woukeu, A. (2005) Managing the Semantic Aspects of Learning using the Knowledge Life Cycle. In Proceedings of The 5th IEEE International Conference on Advanced Learning Technologies (ICALT 2005) (in press), Kaohsiung, Taiwan.

White, S and Davis, H (2000) Creating large-scale test banks: a briefing for participative discussion of issues and agendas, Proceedings of 4th Annual CAA Conference, Loughborough, 2000

Wilson S., Blinco K., and Rehak D (2004) An e-Learning Framework: A Summary. JISC.

Wilson S., Blinco K., \& Rehak D. (2004b) Service-Oriented Frameworks: Modelling the infrastructure for the next generation of e-Learning Systems. JISC 